\title{
From general to specialty hospitals: operationalising focus in healthcare operations
}

\author{
Mandar Dabhilkar ${ }^{1} \cdot$ Anna Svarts $^{2}$ \\ Received: 9 March 2018 / Revised: 12 December 2018 / Accepted: 18 December 2018 / Published online: 7 January 2019 \\ (C) The Author(s) 2019
}

\begin{abstract}
This study proposes an operationalisation of the term focus in healthcare operations. We develop a configuration model consisting of six interrelated dimensions that can be used to characterise hospital focus. The proposed dimensions of focus are Knowledge areas, Procedures, Medical conditions, Patient groups, Planning horizons and Levels of difficulty. The strength of these dimensions is shown through empirical examination, using a three-step methodology to analyse case study data from a unique transformation project where general hospitals were turned into new types of focused specialty hospitals. As our study takes a portfolio approach to the allocation of demand segments to different healthcare delivery units, it contributes to operations management knowledge with a model for segmenting healthcare demand from an operations perspective. This configuration model provides researchers and practitioners with a tool for understanding current configurations as well as for identifying potential new configurations of focus in healthcare.
\end{abstract}

Keywords Focused factory · Health care operations management · Operations strategy · Configuration model · Case research · Stockholm County Council (SLL)

\section{Introduction}

In Sweden, as in many other countries, we are growing accustomed to news stories about healthcare systems with rising costs and a medical profession struggling to meet the everincreasing demands of a growing and ageing population. Focus in healthcare operations is a concept often employed in the debate about how the hospital industry can cut costs and concurrently meet the pressures of increased demand for health services (Bohmer 2009; Christensen et al. 2009; Herzlinger 1997; Porter and Teisberg 2006). The concept of focus was originally coined by Skinner (1974), who discussed possible solutions to the productivity crisis in the North

Mandar Dabhilkar and Anna Svarts contributed equally to the article; names appear in alphabetical order.

Anna Svarts

anna.svarts@indek.kth.se

Mandar Dabhilkar

mandar.dabhilkar@sbs.su.se

1 Stockholm Business School, Stockholm University, SE-106 91 Stockholm, Sweden

2 Department of Industrial Economics and Management, KTH Royal Institute of Technology, SE-100 44 Stockholm, Sweden
American manufacturing industry in the late 1960s and early 1970s. Factories of that time were complex colossi with too many conflicting and varying goals. Skinner argued for more focused factories with fewer demands placed on them, where simplicity and repetition would lead to competitive advantage.

Current general hospitals resemble those complex factory colossi in many respects. Delivering a too broad range of healthcare services leads to conflicting goals at the operational level and consequently results in inferior performance in health outcomes and costs. Therefore, initiatives have begun around the world to create "focused factories" in healthcare. Most of these initiatives are concerned with carving out small parts of hospital operations. These operations can often be easily detached, being independent, planned, repetitive, predictable and with a low degree of patient comorbidity. Hernia repair at the Shouldice clinic is an early example of this (Davidow and Uttal 1989; Heskett 1986). A growing body of literature helps to identify these quick fixes and how the focused factory concept can be implemented in discrete healthcare operations (see, e.g., Bredenhoff et al. 2010).

But what about the hospital operations that remain within the complex colossi after the quick fixes have been taken care of? This study addresses the challenge of dealing with both the often profitable "easy to separate" care and remaining operations. We have studied a unique transformation case of a 
complete healthcare delivery structure in the capital region of Sweden, an attempt that has attained worldwide recognition for its novel portfolio approach to turning general hospitals into new types of (focused) specialty hospitals.

This transformation relates to two key operations management theories: swift, even flow and performance frontiers (Schmenner and Swink 1998). The main idea behind the transformation is to reduce variability at the general hospitals, for example by creating new types of focused healthcare delivery units and relocating care to these, thereby enabling the whole network of hospitals to operate on a better performance frontier. Increased hospital focus in combination with new forms of network integration and coordination will supposedly lead to improved system effectiveness by overcoming trade-offs implicit in the performance frontier curve (Hayes and Pisano 1996).

A managerial problem in the transformation from general to specialty hospitals is to identify alternative specialty hospital configurations. This requires an understanding of how to segment the market for healthcare services from an operations perspective (Berry et al. 1991a). Market segmentation from an operations perspective to evaluate different strategic options is described by Berry et al. (1991b) as "an important prerequisite to the implementation of the focused factory approach". This prerequisite has not been emphasised enough in previous literature on focused factories in healthcare, hence, an operationalisation of focus in healthcare is needed. That is, healthcare requires a configuration model that elucidates interrelated dimensions of focus, thereby helping in decisions regarding allocation of demand for healthcare services to different types of focused healthcare delivery units.

Configuration models that segment market requirements from an operations perspective exist in the operations management literature (see, e.g., Mukherjee et al. 2000, pp. 142143). However, these models were developed for products in a manufacturing setting. Given the unique characteristics of the healthcare industry, implied by the complexity of the human body, the many stakeholders involved in the provision of healthcare and the enormous heterogeneity in the tasks of the medical professions (Boyer and Pronovost 2010), we recommend amending the existing operationalization of focus to better fit healthcare operations. Our overall research question is therefore: Which dimensions of focus are relevant to determine the allocation of demand segments to different healthcare units?

This article shows how the concept of focus in healthcare can be operationalised. We further the ongoing discussion on alternative forms of focus in healthcare (Herzlinger 1997; McDermott and Stock 2011) and propose a configuration model (Boyer et al. 2000; Bozarth and McDermott 1998) consisting of six dimensions of focus. Previous operations management studies on focus in healthcare take fewer dimensions into consideration (e.g. Bredenhoff et al. 2010); we add a more comprehensive perspective to this research. Consequently, the model can be used as a tool for understanding current configurations as well as to identify and explore new configurations of focus in healthcare.

\section{Towards a new set of dimensions of focus in healthcare}

The literature review covers earlier operationalisations of focus as well as empirical studies of focus in healthcare, and is followed by a suggestion of six interrelated dimensions of focus that reflect different principles for limiting the demands that are placed on a healthcare unit.

\subsection{Earlier operationalisations of focus}

The distinguishing characteristic of configuration models is the multidimensional profile that is used to describe organisational, strategic or process types (Bozarth and McDermott 1998). Earlier operationalisations of focus followed one of two parallel research streams that develop Skinner's (1974) ideas into configuration models. The first stream describes specific dimensions of focus with respect to limiting the demands that are placed on a factory. The most complete and recent study in this stream is the work of Mukherjee et al. (2000), who arrive at the following interrelated dimensions of factory focus: Product, Process, Customer market segment, Geographic market region, Volume homogeneity and Suppliers. Mukherjee and his colleagues' configuration model for focusing factories examines a firm's portfolio along these dimensions and then assigns sections of the portfolio to separate factories.

The other research stream develops configuration models that help to assess the degree of focus within a particular site such as one factory or hospital (see, e.g., Bozarth 1993; McLaughlin et al. 1995).

These two streams show that different sorts of configuration models of focus must be developed for different hierarchical levels of an organisation, which is in line with how Johnston (1996) argues focus ought to be achieved in service organisations. At a higher level, models are needed that assign sections of the portfolio of demands to different sites. At a lower level and subsequent stage, models are needed that help to achieve internal strategic fit within each focusing site (Johnston 1996). The current lack of high-level service-based configuration models of focus is a notable gap in the literature; the purpose of this paper is therefore to develop such a high-level model of focus in healthcare. 


\subsection{Empirical studies of focus in healthcare}

As a point of departure, we first define focus in healthcare operations as the differentiation and selection of market segments, followed by the configuration of structural and infrastructural elements of healthcare delivery units to meet the needs of those specific market segments (cf. van Dierdonck and Brandt 1988; McLaughlin et al. 1995).

The empirical studies reviewed mainly concern whether focus has an effect on hospital performance. These are either statistical analyses of large-scale secondary data sources (e.g. Capkun et al. 2012; McDermott et al. 2011) or in-depth case studies (e.g. Pieters et al. 2010; Hyer et al. 2009). The statistical studies mainly concern one type of focus: the selection of a narrow market segment for a service line, such as one diagnosis group or one type of surgical procedure, or in some cases a particular clinical area. They show that this type of focus has an impact on quality in terms of reduced mortality and improved speed in terms of shorter length of stay and lower costs. However, when controlling for factors such as patient comorbidity (Clark 2012) and cherry-picking in terms of selective patient admission in studies of finer granularity (Singh and Terwiesch 2011), diminishing results of focus are found. In addition, one study has shown evidence of complementarities between different service-line segments (Clark and Huckman 2012). This raises concerns about when a narrow service-line focus is actually desirable and about the related need for identifying novel and alternative forms of focus.

The in-depth case studies show that performance improves if focused healthcare units manage to devise and implement a hospital operations strategy that consistently and purposefully adapts structural and infrastructural resource elements, such as hospital layout, technical equipment, care protocols, staff organisation, and financial planning, to prioritised performance objectives (e.g. Hyer et al. 2009). Hospitals need to achieve a fit between their processes and the characteristics of their targeted patient segments (De Regge et al. 2015). Therefore, carving out narrow service-line segments from old general hospitals is not enough; healthcare operations managers in focusing units also have to implement specific focus strategies to improve performance (Peltokorpi et al. 2016).

\subsection{From manufacturing to healthcare services}

Previous research has classified services along dimensions to gain marketing and operational insights (e.g. Silvestro et al. 1992). These dimensions usually describe the degree of customer involvement, service product variety and types of service process with reference to volume per unit and process variety. The dimensions enable distinctions among types of service industries and point out operational implications for service delivery system design. However, they are too broad to single out distinctions within a particular type of industry such as healthcare, and their purpose is not to aid in the allocation of demand segments to different types of focused units.

\subsubsection{Segmentation of demand for providing healthcare}

The most frequently used system to classify healthcare "products" are diagnosis-related groups, developed as a basis for reimbursement (see, e.g., Fetter and Freeman 1986). Recent studies have attempted to segment demand for healthcare provision beyond diagnosis-related groups. One example is Lynn et al.'s (2007) Bridges to Health model, which divides the population into eight groups: people in good health, in maternal/infant situations, with an acute illness, with stable chronic conditions, with a serious but stable disability, with failing health near death, with advanced organ system failure and with long-term frailty. Each group has its own definitions of optimal health and its own service priorities. However, these models do not describe market segmentation from an operational perspective, and they do not aid in the allocation of demand segments to different types of focused healthcare units.

\subsubsection{A new set of dimensions of focus in healthcare}

There are several complementing views in literature on how to limit the demands on healthcare delivery units. We have synthesised these and propose six focusing dimensions for healthcare provision, including a potential measure of focus for each dimension: Knowledge areas, Procedures, Medical conditions, Patient groups, Planning horizons, and Levels of difficulty. For each dimension, a unit with a narrower range of services in this dimension is more focused than a unit with a broader range of services (cf. Mukherjee et al. 2000; Capkun et al. 2012). This definition of focus, as a narrowed range of services, follows the traditional operations management notion of focus, where the narrowing allows for a reduction in the complexity of demands placed on the organization (van Dierdonck and Brandt 1988; McLaughlin et al. 1995).

The meaning of range is closely related to the operations management concept of variety, which implies a negative correlation to volume according to the volume-variety diagonal (Silvestro 1999). Hence, in line with previous research in the area (e.g. Bredenhoff et al. 2010), a narrow range in one dimension of focus, i.e., low variety, usually implies high volume, while higher variety implies lower volume (ceteris paribus).

Under certain circumstances, however, a narrow range in one dimension does not adequately describe a focusing unit's profile. This would be the case for an academic medical centre that chooses to emphasise a smaller set of offerings in a 
particular dimension of focus, while still being mandated to offer a full range of services in that dimension due to its mission. On such occasions "focus as emphasis", conceptualised as a disproportionate emphasis in a dimension of focus, can complement the traditional notion of "focus as narrowing" in the description of the hospital's focus profile (McDermott and Stock 2011).

1) Knowledge areas - This dimension refers to the traditional way of organising healthcare based on the training, skills or experience of physicians and other healthcare professionals. Medical specialty is a related term. The exact medical specialties vary between countries but are often categorised according to a combination of organs, techniques for diagnosis and treatment, and patient age. The Knowledge areas dimension corresponds to product focus in Bredenhoff et al.'s (2010) classification. This dimension may be measured by counting the number of medical specialties represented in the unit, i.e. a lower number of medical specialties corresponds to a higher level of focus.

2) Procedures - This dimension refers to the process or technology used to diagnose or treat patients and is particularly relevant for units specialising in specific types of surgery or specific techniques such as radiotherapy. The Procedures dimension corresponds to process focus in Bredenhoff et al.'s (2010) classification. This dimension may be measured by counting the types of surgical, medical and diagnostic interventions performed at a unit over a certain time period, where a lower number of interventions corresponds to a higher level of focus. Classifications of interventions vary between healthcare systems. However, the World Health Organization is currently developing a global standard, International Classification of Health Interventions (ICHI).

3) Medical conditions - This dimension refers to the disease, injury or other reason that a patient needs healthcare. Care for a single medical condition may require competence from several knowledge areas. Medical conditions is a term proposed by Porter and Teisberg (2006) as a better alternative for segmentation than traditional medical specialties, implying a shift towards more "patient-centric" care. Herzlinger (1997) notes that an effective, focused healthcare delivery unit will contain all the resources required to treat all aspects of the customer's needs; for example, a diabetes-focused facility will also provide nephrologists to manage the kidney disease that often accompanies diabetes. This dimension may be measured by counting the diagnosis codes of a unit's patients over a certain time period, i.e. a lower number of different diagnosis codes corresponds to a higher level of focus. A widely used classification system with diagnosis codes for medical conditions is the International Statistical Classification of Diseases and Related Health Problems (ICD), which is published by the World Health Organization.

4) Patient groups - This dimension refers to patient characteristics of a biological, social or psychological nature that affect the allocation of care, although they are not directly related to the medical condition (Eissens van der Laan et al. 2014; Lynn et al. 2007). Common patient groups are children and the elderly, but groups can also centre on such aspects as ethnicity, occupation or legal status. To measure this dimension it is necessary to understand how patients are grouped in the healthcare system under study (e.g. based on age, gender, source of healthcare financing, ethnicity, occupation or legal status). Measuring the level of focus of a unit involves determining if any patient groups are excluded. As an example, in a study of a healthcare system where some patients are publicly financed, others are covered by private health insurance, and a third category of patients are self-paying, some units may target only one or two of these groups. Treating only one of these categories corresponds to a high level of focus, whereas treating a larger share of patients corresponds to a lower level of focus.

5) Planning horizons - This dimension refers to the predictability of care, typically whether the unit accepts emergency patients, elective patients or both (Joustra et al. 2010). Although often discussed in terms of elective or emergency care, it is a continuous scale - for example, some elective procedures can be planned 6 months in advance, while others need to be scheduled within a few days, reflecting different demands on operations. This dimension can be measured using the categorisation of emergency (immediate need for care), urgent (need care today), semi-urgent (need care within 1 to 2 weeks, also referred to as semi-elective), and elective patients (see, e.g., Zonderland et al. 2010). Treating only one of these categories corresponds to a high level of focus, while treating more categories corresponds to increasingly lower levels of focus.

6) Levels of difficulty - This dimension refers to the extent advanced competence or technology are needed in healthcare delivery. Reasons healthcare might require more advanced resources include the severity of the illness, in terms of the medical condition itself and the complication level of the individual's medical condition, or patient risk factors (e.g. comorbidity, obesity or a 
history of heart problems). Another reason could be a low degree of standardisation of diagnosis and treatment, implying a greater need for advanced capabilities. Christensen et al. (2009) refer to aspects of this type of care as 'intuitive medicine'. Similarly, Bohmer (2009) uses his iterative versus sequential dichotomy to single out this kind of care. Finally, Herzlinger (1997, p. 188) notes that "most focused factories will serve the needs of most of us, but a few will specialize in very complex cases". Similar to the Patient groups dimension, the measurement of this dimension requires an understanding of the specific healthcare system being studied and how level of difficulty is classified in that system. Generally, levels of difficulty can be measured using the broad categories of primary, secondary, tertiary and quaternary care. Only one category of care in a unit (e.g. only primary care) corresponds to the highest level of focus, while having all four categories corresponds to low focus.

This last dimension needs to be described at a particular moment in time, since the perceived difficulty of medical conditions and procedures is constantly changing. Typically, new diagnostic or surgical procedures require close collaboration with researchers or other experts. This means that the actual services offered by a clinic focused on a high level of difficulty will change over time, as procedures become commoditised and are transferred to other clinics or to primary care (Christensen et al. 2009).

The six interrelated dimensions of focus form a multidimensional construct of profile model-type. As such, every representation of a focusing healthcare delivery unit will be formed as a combination of its dimensional characteristics (Law et al. 1998, p. 744). The combinations of dimensional characteristics can be graphically represented in a chart, such as the spider chart in Fig. 1. Values closer to the centre of the chart represent a higher focus (i.e., a narrower range of services) and values closer to the edges represent a lower focus (i.e., a broader range of services) in that dimension of focus.

\section{Research methodology}

We use a case study of a healthcare authority to develop and validate our high-level configuration model, which we argue is one important prerequisite for the implementation of focus in healthcare delivery network structures (Berry et al. 1991b). Case research is suitable for theory extension (Voss et al. 2002), and our study aims to extend theory through what Ketokivi and Choi (2014) refer to as 'theory elaboration', which "focuses on the contextualized

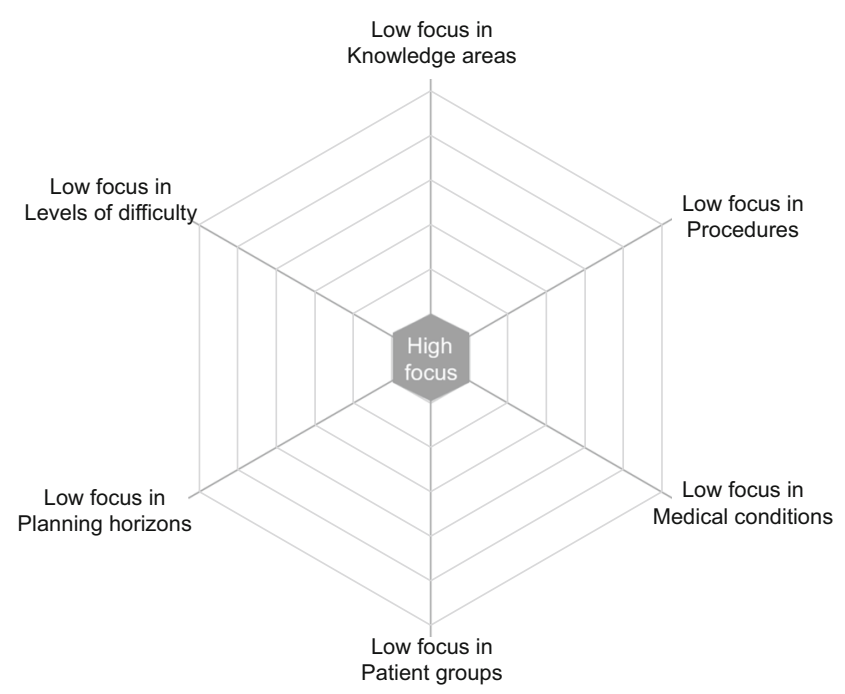

Fig. 1 Profile model of focus in healthcare

logic of a general theory" (p. 236). In this study we translate and further develop Mukherjee et al.'s (2000) configuration model of manufacturing factory focus in the context of healthcare.

Using existing operationalisations of focus as the point of departure and comparing these to hospital configurations found in our case study, we have developed a proposal for an operationalisation of focus in healthcare.

At the onset of the study we had an interest in how and why healthcare organisations chose to establish 'focused factories'; hence, we studied literature on the Shouldice hospital and similar clinics that focus on a narrow set of knowledge areas and procedures and on performance effects of focused factories in healthcare. During the interviews we realised that when looking at healthcare provision for a whole region, hospital focus appeared more multifaceted than what was reflected in the literature, and that a high-level multidimensional construct of focus in healthcare was called for.

We then returned to the literature to see how different types of focus are described (e.g. Bredenhoff et al. 2010). Comparing this to our case, we developed a preliminary draft of focus dimensions in healthcare. We then performed an extensive literature search about focus operationalisations (e.g. Mukherjee et al. 2000) and different interpretations of focus in healthcare (e.g. van Dierdonck and Brandt 1988; McDermott and Stock 2011; McLaughlin et al. 1995), to refine the framework into the configuration model proposed here. This interplay of theory and empirics is in line with Ketokivi and Choi's (2014) recommendations for theory elaboration using an abductive reasoning approach and assertion that 
"successful theory elaboration hinges on the researcher's ability to investigate the general theory and the context simultaneously, in a balanced manner" (p. 236).

Although the configuration model was developed iteratively between existing theory and empirical context, we need a structured approach to ensure that the proposed framework is consistent with our empirical data. Hence, in the subsequent data analysis we used a threestep process to validate the relevance of our proposed operationalisation in the context of our specific empirical setting.

\subsection{Selecting the case}

This study concentrates on healthcare administered by Stockholm County Council (SLL for its name in Swedish, Stockholm läns landsting), the public health authority in the Swedish capital. The study object was chosen due to the transformation of healthcare delivery currently taking place. In this transformation project, the health authority faced the managerial problem that this study addresses: how to relocate hospital operations to new types of specialty hospitals from existing general hospitals. The case is an exceptional opportunity to study focus in healthcare operations, since the change project is so comprehensive, concerning the whole portfolio of healthcare delivery in the region, rather than just a specific hospital.

Sweden is a good country for this type of healthcare operations strategy study, for two main reasons. Firstly, although there are many areas for improvement, the Swedish healthcare system is often ranked as one of the best in the world (Davis et al. 2014; OECD 2013) and is considered a model for other countries to emulate due to excellent health outcomes and well-developed strategies to improve quality of care (OECD 2013). Secondly, the Swedish system, with a single health authority that is responsible for healthcare provision to all inhabitants in a region, is well suited for studying the design of a comprehensive healthcare delivery structure.

The uniqueness of the case motivates our choice of a single case study. Although multiple cases can make research more robust, single cases can also be used if they are unique exemplars or opportunities for unusual research access (Barratt et al. 2011). We believe both conditions are fulfilled in this case.

Objects of analysis in the case study are the units in the healthcare delivery structure; i.e. hospitals and clinics, and the segments of patient demand allocated to those units.

\subsection{Data collection}

Twenty-six interviews make up the most important part of the data collection. The interviewees, all participants in an SLL project for designing new elective specialty care units, include both supply-side representatives such as hospital managers and demand-side representatives from the county council. The interviewees represent a total of 17 different clinics and organisations (see Table 1).

The interviews followed an interview protocol that included questions about the transformation project and the allocation of healthcare services to different clinics, such as "Which care should be conducted at the new specialty care units?" "Why?" "Is there any type of care that would be inappropriate for the new specialty care units?" "Why?" The interviewees were encouraged to discuss principles for allocation as well as give specific examples (summarised in Table 2). Interviews were scheduled for $90 \mathrm{~min}$ each; actual interview time varied between 30 and $120 \mathrm{~min}$. New interviews were booked until we experienced saturation in the sense that each interview was providing little additional information.

The study also included 16 one-to-two-hour meetings with a key informant, a project manager at SLL. To achieve triangulation of sources whenever possible, multiple secondary data sources were used, such as publicly available reports and internal documents (memos, meeting minutes, etc.). Data triangulation in terms of multiple sources of evidence is believed to improve validity in case research and provide stronger substantiation of constructs and propositions (Voss et al. 2002). The meetings with our key informant were important in this respect, since the informant helped us find additional sources to challenge or support emerging findings from the interviews. We let the key informant and another SLL representative review the draft case study report to make sure that the documentation of case data was accurate (Voss et al. 2002, p. 212). The same SLL representatives, together with the chief medical officer from one of the hospitals, reviewed the tentative framework with the six dimensions of focus. This review was a measure to improve truth value by testing findings against the group from which the data were drawn and people familiar with the phenomenon being studied (Krefting 1991, pp. 215-216).

The main part of the data collection was carried out at an early stage of the transformation project, after the decision to change the healthcare delivery structure was made and the goals were set but before implementation had started. While we interviewed project participants and held regular meeting with the project manager, they worked to identify ways to meet the ambitious project targets by setting guidelines for the transformation, setting new roles for healthcare units and setting up pilot implementations. The project is currently finalising the implementation phase, and we have continued to complement the study with emerging data about the healthcare units in the new structure (see Appendix B - Focus assessment of three example units). 


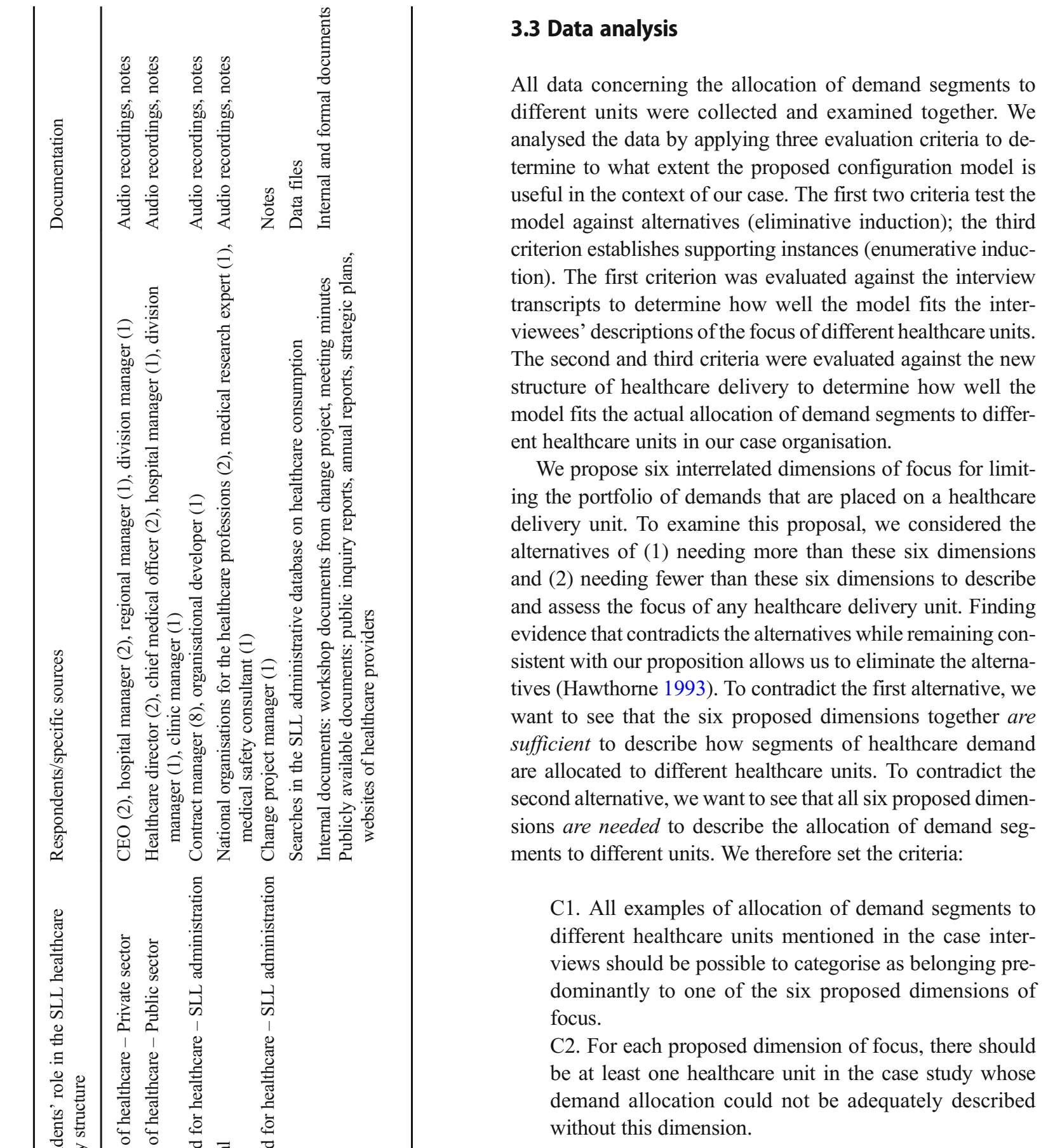

Miles et al. (2014) suggest that eliminative induction should be combined with enumerative induction, the method of collecting a number and variety of instances that agree. We sought supporting instances for the usefulness of our model by examining if our model can be applied to the new configurations of specialty hospitals in our healthcare delivery transformation case. To do this, we set the additional criterion:

C3. It should be possible to apply the operationalisation of focus to each new configuration of specialty hospital in 
Table 2 Display of interview responses on allocation of care to different units

\begin{tabular}{|c|c|c|c|}
\hline $\begin{array}{l}\text { Dimension of focus } \\
\text { in healthcare } \\
\text { operations }\end{array}$ & $\begin{array}{l}\text { Corresponding class/ } \\
\text { category }\end{array}$ & $\begin{array}{l}\text { Frequency of } \\
\text { instances across } \\
\text { the } 26 \text { interviews }\end{array}$ & Examples of quotes \\
\hline Knowledge areas & $\begin{array}{l}\text { Examples of medical } \\
\text { specialties and parts of } \\
\text { medical specialties }\end{array}$ & $16 / 26$ & $\begin{array}{l}\text { "orthopaedics", “geriatrics”, "psychiatry”, "gynaecology”, "cardiology”, } \\
\text { "urology" }\end{array}$ \\
\hline Procedures & Examples of procedures & $9 / 26$ & $\begin{array}{l}\text { "day surgery", "radiation therapy for cancer", "endoscopy", "melanoma skin } \\
\text { cancer screening", "outpatient care", "advanced medical aftercare" }\end{array}$ \\
\hline Medical conditions & $\begin{array}{l}\text { Examples of diseases, } \\
\text { injuries and other } \\
\text { medical conditions }\end{array}$ & $12 / 26$ & $\begin{array}{l}\text { "breast cancer centre covering the entire chain of care", "chronic diseases", } \\
\text { "dementia", "illnesses that require 24-h access to operating room } \\
\text { resources, such as stomach pain", "cancer", "diabetes" }\end{array}$ \\
\hline Patient groups & Examples of patient groups & $3 / 26$ & "multi-ethnic centre", "elderly patients", "inpatient care for children" \\
\hline Planning horizons & $\begin{array}{l}\text { Planned/elective vs. acute } \\
\text { care }\end{array}$ & $16 / 26$ & $\begin{array}{l}\text { "elective care", "mainly planned care", "emergency care", "no emergency } \\
\text { care but acute same-day appointments" }\end{array}$ \\
\hline Levels of difficulty & $\begin{array}{l}\text { Examples that state a level } \\
\text { of medical complexity } \\
\text { and/or a need for ad- } \\
\text { vanced resources }\end{array}$ & $18 / 26$ & $\begin{array}{l}\text { "the small group of patients with comorbidity", "only patients with ASA } \\
\text { [American Society of Anesthesiologists] physical status } 1 \text { and 2", "low } \\
\text { complexity in terms of few additional services required and no need of } \\
\text { resources such as an intensive care unit", "no need for access to an } \\
\text { intensive care unit", "care with high degree of specialisation, e.g. that } \\
\text { which is very seldom performed", "care with no need for special technical } \\
\text { resources, e.g. linear accelerators" }\end{array}$ \\
\hline
\end{tabular}

the case study by assessing the degree of focus in the six proposed dimensions.

\section{Results}

The case organisation, Stockholm County Council (SLL), is responsible for the provision of healthcare to the inhabitants of the capital region of Sweden. They administrate and through taxes finance the region's healthcare ('demand-side organisation') and deliver healthcare via a large number of primary care centres, private clinics, local hospitals and six former general hospitals ('supply-side organisation'). A new hospital, New Karolinska Solna (NKS), with fewer inpatient beds, no regular emergency department (but an intensive emergency care department) and a main focus on tertiary care, is replacing one of the general hospitals. Appendix A provides more information about the SLL healthcare delivery structure.

\subsection{Project context and new hospital configurations}

SLL forecasts a sharp increase in the need for healthcare over the next decades, based on the rapidly growing and ageing population in the region. The county council's projected income from taxes is not increasing proportionally to the healthcare demand. Hence, SLL concluded that just expanding the current healthcare delivery network structure to meet the projected capacity shortage, for example by adding a new general hospital, was not a viable solution. Rather, they needed to transform healthcare delivery in the region in a radical way to enable the whole network of healthcare delivery units to operate on an improved performance frontier. That is, to improve cost efficiency while meeting the demand for high-quality and accessible care. In 2011, SLL launched a project to plan and implement this transformation and explicitly defined three performance objectives for the future healthcare delivery structure: high accessibility, high quality and patient participation, and high efficiency. The main supposed mechanism to reach these goals was to assign new roles for current and future hospitals in the healthcare delivery structure. SLL's analysis showed that the unit cost for treatment at general hospitals was substantially higher than the cost for the same treatment outside general hospitals, due to expensive technology and on-call specialists. Therefore, they identified relocation of patients to the right level of care - with patients needing only emergency hospital resources located to those hospitals, and other patients located to different types of healthcare units - as a means to achieve highquality, accessible care while simultaneously improving cost efficiency. This included a shift from inpatient to outpatient care, treating a larger share of patients at other healthcare providers outside the traditional general hospitals, and last, sending a larger share of patients to finish the care episode outside the traditional general hospitals with other types of healthcare delivery units or even at home.

Part of the project was the development of new elective specialty care units. The idea was to move elective care that does not require emergency hospital resources (such as proximity to an intensive care unit) from general hospitals to the new elective specialty care units and to other private clinics. 
This allows the general hospitals to focus on secondary emergency care, although they retain certain more difficult or risky elective procedures. Care for the most complicated or unusual conditions, care requiring special competence or special resources, as well as new and experimental procedures (together referred to as 'highly specialised care' by SLL) will be moved to NKS. Most emergency care that cannot be covered by primary care providers remains at the former general hospitals, which we now call 'emergency care units'.

In sum, three new configurations of specialty hospitals are emerging within SLL:

(1) NKS, which is a highly specialised care unit,

(2) elective specialty care units that accommodate nonurgent elective care being relocated from former general hospitals and

(3) former general hospitals that are transformed into emergency care units for secondary care.

Three SLL units serve to illustrate the new configurations of specialty hospitals. The first is NKS, the highly specialised care unit. The second is Sabbatsberg, an elective specialty care unit. The third is Danderyd hospital, a general hospital that is becoming more of a pure emergency care unit.

NKS's profile is healthcare that is highly specialised or requires large investments in technology or close collaboration in research. The new university hospital will have 6000 employees. NKS will not have an open emergency room but will receive emergency patients who are triaged for the hospital's specific resources, such as patients from ambulance transport, helicopter transport or other units.

Sabbatsberg has been chosen as a pilot unit for elective specialty care. The unit has 170 employees. Current specialties include orthopaedics, hand surgery, general surgery, urology, gynaecology, otorhinolaryngology, ophthalmology, cardiology and breast cancer. An expansion of the unit's operations is part of the project; SLL has proposed a profile for Sabbatsberg with a focus on elective care where the risk of needing intensive care or other hospital resources is low. Most elective specialty care units cover only one medical specialty, but Sabbatsberg is an example of a clinic that provides elective and low-risk care while covering several medical specialties.

Danderyd hospital has traditionally been a general hospital with all major medical specialties handling all patients. With 3700 employees, Danderyd hospital has a large maternity clinic, a large volume of emergency care, and a large amount of outpatient care. Population growth and the highly specialised care profile of NKS mean that the other SLL hospitals need to handle a dramatic increase in the number of emergency patients.
Healthcare operations regarded as highly specialised are transferred to NKS. Less advanced elective care, such as less complicated surgery and return visits, are transferred to the new elective specialty care units. As a result of these changes, Danderyd hospital now has an explicit focus on standard emergency care processes and follow-up inpatient care.

Following from the hospitals' new focusing profiles, structural and infrastructural elements of their respective operations strategy vary with configuration type. At NKS and Danderyd, for example, where the implied demand uncertainty is high due to their focus on tertiary and/or emergency or urgent care, hospital layout, medical technology and aggregate capacity planning are designed to cope with higher process variety and variation. At Sabbatsberg, on the other hand, where the implied demand uncertainty is much lower due to their focus on elective care in a few medical conditions that do not need emergency hospital resources, clinical layout and medical technology are more dedicated to the mainly standardised processes in targeted medical conditions.

\subsection{Evaluation of C1}

The first evaluation criterion is: All examples of allocation of demand segments to different healthcare units mentioned in the case interviews should be possible to categorise as belonging predominantly to one of the six proposed dimensions offocus. The key outcome of this evaluation is whether there are any examples of focus mentioned by the interviewees that fall outside of our proposed dimensions, thus indicating that some aspect of focus is not covered by our configuration model.

To evaluate this, we compiled all instances where interviewees mentioned allocation of care to different units. This compilation resulted in a list of 84 quotes, summarised in Table 2. For each quote we then asked, "what is this specific thing an instance of?", to compare the particular demand segment in the quote with the six proposed dimensions of focus used as abstractly defined classes (Miles et al. 2014, p. 285).

Some quotes could possibly be interpreted as belonging to more than one class, which is natural since the dimensions are interrelated, but there was no example that fell outside of the predefined classes. Thus, Table 2 shows that all examples of demand allocation are covered by the six proposed dimensions of focus, and the first evaluation criterion is therefore fulfilled.

\subsection{Evaluation of C2}

The second evaluation criterion is: For each proposed dimension of focus, there should be at least one 
healthcare unit in the case study whose demand allocation could not be adequately described without this dimension.

To evaluate this we looked at the total SLL healthcare delivery structure, the whole portfolio of healthcare delivery units. Thus far we have only discussed the three new configurations of specialty hospitals. These configurations represent a large share of all healthcare delivery within SLL, but they are complemented by many other configurations of specialty hospitals, local hospitals, clinics and doctors' practices. Some of these represent traditional outpatient healthcare services, such as ENT (ear, nose and throat) practices run by individual otorhinolaryngologists; others are results of previous attempts to shift care from general hospitals to less costly facilities such as specialised orthopaedic clinics.

The three new configurations presented in section 4.1 are not sufficient to motivate all six dimensions of our proposed configuration model. The reason is that Planning horizons and Levels of difficulty were the guiding principles in designing the three configurations under scrutiny in the transformation project we studied. Hence, to find examples of healthcare units that cannot be adequately described without the dimensions of Knowledge areas, Procedures, Medical conditions and Patient groups, we have to look beyond those three new configurations. From the wider set of all existing configurations we selected the examples shown in Table 3. (Note: Stockholm South General Hospital, which is given as an example for the Planning horizons dimension, is one of the new emergency care units, with a configuration similar to Danderyd hospital.) After the six unit names and descriptions, Table 3's third column pinpoints the key principle for demand allocation to each unit; the last column identifies the most relevant dimension of focus. Table 3 shows that for each of the six proposed dimensions of focus, there is at least one healthcare unit within SLL whose demand allocation could not be adequately described without this dimension. Hence, the second evaluation criterion is fulfilled.

\subsection{Evaluation of $\mathrm{C} 3$}

The third evaluation criterion is: It should be possible to apply the operationalisation of focus to each new configuration of specialty hospital in the case study by assessing the degree of focus in the proposed dimensions.

To evaluate this, we applied the proposed dimensions of focus to the three units introduced in section 4.1 by assessing the degree of focus in each dimension. For each unit the range of services or emphasis has been assessed for all dimensions, based on interviews with hospital management and on searches in the county 
council administrative database on healthcare consumption, where details on patients' diagnoses and procedures are recorded by all units.

Results are depicted in Fig. 2, and details of the assessments are provided in Appendix B. As earlier explained, values close to the centre of the chart represent a higher degree of focus, while values further out represent a lower degree of focus. Hence, a traditional general hospital providing a complete range of services in all dimensions would have a profile along the outer edges of the chart (low focus in all dimensions).

Figure 2 shows that the three units have very different focus profiles. The most focused unit in all dimensions is the elective specialty care unit (Sabbatsberg), consistent with their mission of providing specific types of elective care only. The highly specialised care unit (NKS) and the emergency care unit (Danderyds sjukhus) have lower degrees of focus than Sabbatsberg, consistent with their broader missions. However, these two hospitals also show higher degrees of focus than a traditional general hospital with a complete range of services would, and moreover, their emphasis on highly specialised care and emergency care, respectively, can be discerned.

NKS has their highest degree of focus in the Levels of difficulty dimension, consistent with its explicit profile of highly specialised care. The streamlining of the hospital, in terms of transferring highly specialised care from other hospitals to NKS and moving less advanced care from NKS to other hospitals, is an ongoing process. Hence, if this assessment is repeated at a later time, the result will most likely be an even higher degree of focus in the Levels of difficulty dimension. NKS has a lower degree of focus in Procedures, due to performing a wide but not full range of surgical, medical and diagnostic interventions (patients needing less advanced interventions are referred to other units). NKS has their lowest degree of focus in Knowledge areas, Medical conditions, Patient groups, and Planning horizons, since they employ physicians from most medical specialties, treat a wide range of diagnoses (when the severity of illness for the individual patient requires the hospital's advanced resources), target the whole population in the region through the public health care system, and have large volumes of both emergency and elective care. However, in 2018 the general emergency ward at NKS is closing and only emergency patients in need of highly specialised care will be brought to the hospital by ambulance or referred from other hospitals. Hence, a higher degree of focus in the Planning horizons dimension is to be expected if this assessment is repeated for NKS at a later time, because the hospital is moving towards a distinct elective care profile.

Sabbatsberg has the highest degree of focus in the Planning horizons and Levels of difficulty dimensions, since they exclusively provide elective care that doesn't require highly specialised resources or capabilities. Sabbatsberg also has a high degree of focus in Knowledge areas, Procedures, and Medical conditions, since they provide a limited range of services in terms of interventions and diagnoses and consequently only need physicians from a limited range of medical specialties. Sabbatsberg has their lowest degree of focus in the Patient groups dimension, since they provide most of their healthcare services through the public healthcare system, although a third of their services target only the group of patients that have private medical insurance (6\% of the population in Sweden) or are able and willing to pay out-of-pocket.

Danderyd hospital has their highest degree of focus in the Planning horizons dimension, consistent with their profile as an emergency care hospital. Eighty-two percent of Danderyd's admissions are emergencies, and this
Fig. 2 Example of focus assessment in healthcare delivery

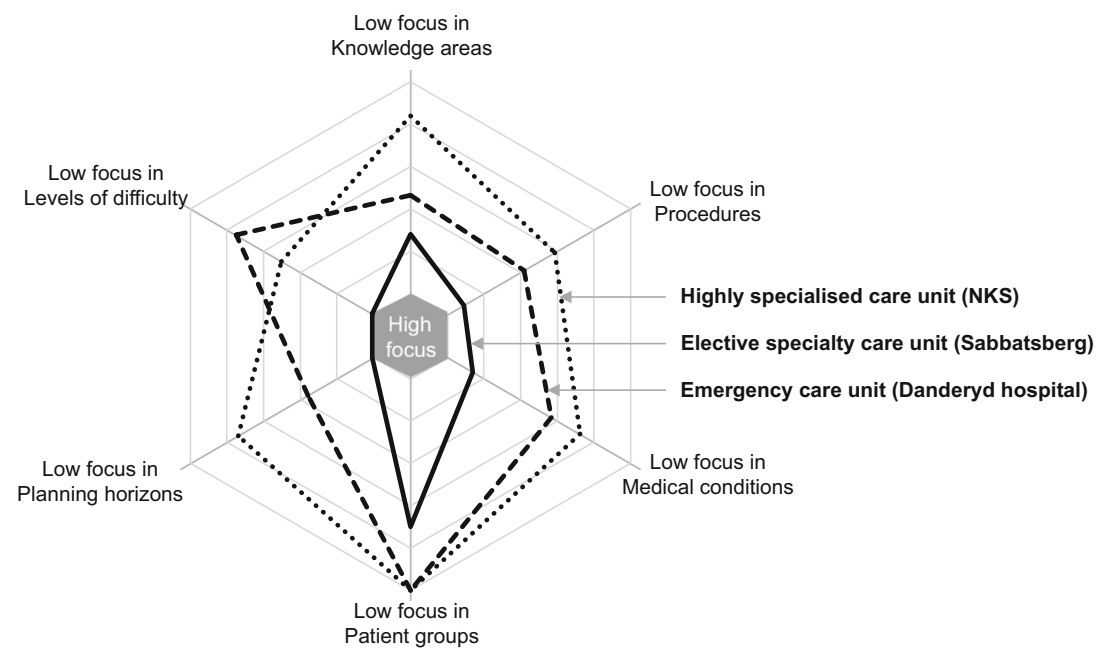


number has been increasing over the last years as elective care has shifted to elective specialty care units and (for highly specialised care) to NKS. This in an ongoing development, although the hospital plans to retain a small share of elective care that is needed to provide sufficient experience and training for physicians and nurses. Danderyd hospital has lower degrees of focus in Knowledge areas, Procedures, Medical conditions, and Levels of difficulty. This reflects that the hospital employs a relatively wide range of medical specialties and can treat most diagnoses and perform many interventions as required by an emergency hospital. However, the more unusual medical specialties have been transferred to NKS and therefore Danderyd hospital has a higher degree of focus in e.g. Knowledge areas compared to NKS. Danderyd hospital is less focused than NKS in the Levels of difficulty dimension, since they perform some highly specialised care while having an overall emphasis on standard secondary care. Danderyd hospital has a low degree of focus in Patient groups since they target the whole population in the region through the public health care system.

When assessing the degree of focus of the three example units, we applied the operationalisation of focus to each new configuration of specialty hospital in the SLL case. This assessment, done using data from the case study, implies that the third evaluation criterion is fulfilled.

\subsection{Summary of results}

We have demonstrated the explanatory power of our proposed configuration model by showing that the actual patterns of data in our case study match the proposed model. Firstly, all examples of demand allocation mentioned in the interviews are consistent with the model (Table 2). Secondly, for each dimension in the model, there is an example of a healthcare delivery unit whose focus profile is strongly related to this specific dimension (Table 3). Finally, the degree of focus and differences between units in the proposed dimensions can be assessed for the three hospitals that represent the new types of focused healthcare units identified in the case study (Fig. 2; Appendix Tables 4 and 5).

\section{Discussion}

This study contributes to knowledge by extending the work of Mukherjee et al. (2000). We translate their notion of dimensions of manufacturing factory focus into the context of healthcare delivery. In doing so we continue the discussion that Herzlinger (1997) and McDermott and Stock (2011) initiated on alternative forms of focus in healthcare to meet the ever-increasing pressures on the sector.

While Mukherjee and colleagues list Product, Process, Customer market segment, Geographic market region, Volume homogeneity and Suppliers as dimensions of focus, we have found another set more relevant for healthcare operations: Knowledge areas, Procedures, Medical conditions, Patient groups, Planning horizons and Levels of difficulty. For some dimensions the resemblance is high between the two sets, for some the resemblance is low due to the healthcare context, as we discuss below.

Product and Process in manufacturing resemble Knowledge areas and Procedures in hospital operations; this is also how Bredenhoff et al. (2010) assess focus in healthcare. Customer market segment in manufacturing resemble both Medical conditions and Patient groups in hospital operations. The dimension Medical conditions represents the medical aspect of the customer (patient) market. Segmentation based on medical conditions is gaining attention worldwide as a more patient-centric alternative to traditional knowledge areas (medical specialties) (cf. Porter and Teisberg 2006). The dimension Patient groups, on the other hand, relates to patients' demographic characteristics and represents the nonmedical aspect of the customer (patient) market. These nonmedical aspects are present in a population and affect segmentation and allocation of care, although they are not directly related to the medical condition itself (Lynn et al. 2007).

The next three manufacturing dimensions in Mukherjee et al.'s (2000) model, i.e., Geographic market region, Volume homogeneity and Suppliers, do not correspond well to the new dimensions we propose for healthcare, at least not directly.

Geographic market region is a highly relevant focus principle in manufacturing. Different geographic market regions often require specific configurations, which affects the requirements placed on operations; for example due to technological standards, regulatory differences, and delivery, shipping and packaging requirements. These aspects have not been found as important in the healthcare context where patients typically seek care locally or nationally.

One geographic aspect that has been found important to consider in healthcare, though, is geographical proximity to care. However, neither our data analysis nor our literature review support geographical proximity to care as a separate dimension of focus in healthcare. Rather, it is an important aspect within other dimensions. One 
example is the Medical condition focus. As Porter and Teisberg explain, hospitals that focus on just one medical condition, such as prostate cancer, through the creation of integrated practice units will require high volumes (in terms of scale) in order to cover costs for this organisational form. As volumes will be concentrated to these fewer units, patients will naturally have to travel longer distances. Another example where geographical proximity is embedded within other dimensions is emergency care, which we have within the focus dimension Planning horizons. Emergency care has to be located within a certain geographical proximity in order to serve its purpose and fulfil its goals.

Volume homogeneity is the second dimension of manufacturing focus that doesn't correspond well to the healthcare context. This is in contrast to the concept of scale, which is very relevant not only in manufacturing but also in healthcare, as mentioned previously. In manufacturing, a fundamental idea is to assign production of products of similar order sizes and batch sizes to certain plants of the firm (Mukherjee et al. 2000). Volume homogeneity does not play the same role in healthcare, where the batch size is usually one, with every patient providing significant self inputs into the service production process (Sampson and Froehle 2006, p. 332). However, it is still desirable, of course, to reduce variability in healthcare operations processes, which is accomplished through reducing variability in customer inputs. A focused hospital limits the range of service offerings by limiting the range of customer self inputs in one or several of the six proposed dimensions of focus.

Lastly, the manufacturing dimension called Suppliers does not correspond well to the healthcare delivery context. Healthcare units are seldom dependent on close proximity to suppliers, unlike in manufacturing, where suppliers are often located close to their customers' final assembly lines due to just-in-time practices.

So instead of the manufacturing dimensions Geographic market region, Volume homogeneity and Suppliers, we propose Planning horizons and Levels of difficulty as more relevant to the healthcare context. Regarding Planning horizons, urgent cases are traditionally treated in emergency departments, separately from cases that can wait (Hopp and Lovejoy 2012). The SLL case shows how this tradition in medicine is taken further by the creation of new emergency care units. The Levels of difficulty dimension reflects different types of medicine (e.g. intuitive vs. empirical) and their respective operational implications (Christensen et al. 2009). The SLL case illustrates how distinguishing among these different types of medicine results in increased focus also for nonstandard, unpredictable healthcare operations.

\section{Conclusion}

We conclude that focus in healthcare operations can be operationalised as a configuration model consisting of the interrelated dimensions Knowledge areas, Procedures, Medical conditions, Patient groups, Planning horizons and Levels of difficulty. For each dimension, a healthcare unit with a narrower range of services in this dimension is more focused than a unit with a broader range of services.

The study helps healthcare practitioners make more informed decisions, in that our configuration model can be used as a tool to understand configurations of focus in current hospitals as well as to identify options for new configurations of hospital focus. In addition, the case provides valuable insights from a pioneering transformation project of a complete healthcare delivery network structure.

Taking a portfolio approach to the allocation of demand segments to different healthcare units, the study contributes to knowledge with a configuration model for segmenting healthcare demand from an operations perspective. This allows future research about focused factories in healthcare to take the whole healthcare delivery structure into account to optimise system performance, rather than concentrating on only one type of focus (such as focus on a narrow set of knowledge areas and procedures, as exemplified by the Shouldice clinic's focus on hernia repair). Examples of possible measures along each dimension of focus are also proposed to support future research.

Given that our configuration model is informed by and developed vis-à-vis current literature, it is likely to be applicable to healthcare operations in general. However, a key limitation of our study is that the model development and empirical examination uses data from one region and within one national healthcare system. We have therefore attempted to include enough information about the context of the case to make it possible for readers to judge the transferability of the results to other healthcare settings.

It would be very interesting to know if SLL's transformation of its healthcare delivery structure leads to an improved performance frontier. However, at this point in time it is too early to assess such performance effects. We look to future research to shed light on the performance effects, presumably by employing a quantitative research design to assess the outcome of relevant performance objectives. 
We see a number of areas for further research. Our work shows that there are more types of hospital focus than earlier studies recognise; these new types of focus call for additional studies. Since focus per se does not lead to improved performance, specific configurations of structural and infrastructural operations strategy elements ought to be developed for the new types of focused healthcare units. Also, as mentioned above in relation to SLL, analyses of performance effects of focus-based transformations of healthcare delivery structures on individual hospitals as well as on the system as a whole is an intriguing area for further research. The configuration model proposed in this paper could be used as a stepping stone in such a research endeavour.

\section{Appendix A}

\section{Stockholm County Council (SLL) healthcare delivery structure}

In Sweden, publicly financed healthcare (98\% of all healthcare) is the responsibility of 20 county councils. The Stockholm County Council (SLL for its name in Swedish, Stockholm läns landsting) administers the largest county, with a population of 2.2 million. The healthcare system is mainly tax-funded. SLL is responsible for the provision of all healthcare to all inhabitants, which translates to around 350,000 hospital admissions, 8 million visits to doctors, and 9 million visits annually to other healthcare professionals such as district nurses. SLL has separate organisations for administration and financing healthcare ('demand side') and for healthcare delivery ('supply side'), organised as a large number of primary care centres, private clinics, local hospitals and six former general hospitals. In total, SLL has around 1000 contracted healthcare providers. Measured in share of publicly financed healthcare cost, private healthcare providers, including one of the former general hospitals, deliver $60 \%$ of primary care and $20 \%$ of specialty care. The new university hospital that is replacing one of the general hospitals, New Karolinska Solna (NKS), is built with a public-private partnership model.

\section{Appendix B}

\section{Focus assessment of three example units}

Table 4 provides data on the range of services in each focus dimension for the three sample units in the SLL case study. The first row, Knowledge areas, shows the number of medical specialties among the employed physicians in each unit (for NKS, 43; for Sabbatsberg, 13; and for Danderyd hospital, 21). The same row shows the minimum potential number of medical specialties (1), i.e. the number for a hospital where all employed physicians belong to the same medical specialty, and the maximum potential number (44), i.e. the number for a hospital that employs at least one physician from each of the medical specialties listed by the Swedish National Board of Health and Welfare. The minimum value represents the highest possible focus; the maximum value represents the lowest.

The second row, Procedures, shows the number of unique codes for surgical, medical and diagnostic interventions that were recorded at the unit over a one-year period (for NKS, 4160; for Sabbatsberg, 644; and for Danderyd hospital, 2982). For this dimension the maximum number is defined as the number of unique codes that were recorded at all SLL healthcare units during the same time period (7113).

The third row, Medical conditions, shows the number of unique codes for patients' main diagnosis that were recorded at the unit over a one-year period (for NKS, 6119; for Sabbatsberg, 1189; and for Danderyd hospital, 4804). As for the previous dimension, the maximum is defined as the number of unique codes that were recorded at all SLL healthcare units during the same time pe$\operatorname{riod}(8439)$.

The fourth row, Patient groups, shows the share of privately funded patients (for NKS, 0\%; for Sabbatsberg, 30\%; and for Danderyd hospital, $0 \%$ ). This is based on the finding that the main differentiator between the analysed clinics in the Patient groups dimension is source of funding (in another context it could be more relevant to base the assessment on age groups targeted by different units). Degree of focus in this dimension could be measured by counting the number of different patient groups in terms of funding, i.e. one group for NKS and Danderyd hospital (public patients) and two groups for Sabbatsberg (public and private patients). However, private medical insurance is unusual in Sweden, and the absolute majority of all patients seek healthcare through the public (tax-funded) healthcare system ( $98 \%$ of all healthcare consumption). We therefore view units that accept public patients as unfocused and units that specifically target the small privately funded patient group as focused. In some cases, such as Sabbatsberg, some of the hospital's clinics are open for public patients whereas other clinics are for private patients, and to reflect this, we measure the degree of focus as the percentage of privately funded patients. The 
minimum value, representing the highest possible focus, is then defined as $100 \%$ and the maximum value, representing the lowest possible focus, as $0 \%$.

The fifth row, Planning horizons, shows the units' emphasis on either elective care or emergency care. Degree of focus in this dimension could be measured in terms of narrowing the range of services - in this case Sabbatsberg would have a high degree of focus, since they only have elective care. While correct, we believe that this way of measuring is less meaningful for large hospitals that are required to always have at least a small amount of both elective and emergency care. Yet the difference in demands placed on operations from a 50 50 split of elective and emergency care, compared to a 95-5 split, is vast. Our interviewees have witnessed the difficulties of combining the two planning horizons when faced with large volumes of both elective and emergency care, e.g. in surgery scheduling. To capture this aspect we instead measure focus as emphasis on either category in this dimension. For Sabbatsberg, the table shows the unit's emphasis on elective care (100\%). For NKS and Danderyd hospital, the table shows each unit's emphasis on emergency care (for NKS, 30022 emergency admissions of 47,341 total admissions results in $63 \%$ emergency care; for Danderyd hospital, 37,787 of 45,805 admissions results in $82 \%$ emergency care). Based on the interviews, we believe that the calculated values for emergency care at these two units are probably somewhat low. The reason is that some patients that seek urgent care are eventually admitted as elective patients. As an example, a patient with a leg fracture who is received in the emergency room is often sent home after diagnosis and scheduled for surgery later in the same week, which is then recorded as elective care. The minimum value, representing the highest possible focus, is defined as $100 \%$; the maximum value, representing the lowest possible focus, as $50 \%$ (i.e. an even split between the two different planning horizons).

The sixth row, Levels of difficulty, shows each unit's emphasis on secondary care or tertiary care. Degree of focus in this dimension could be measured by counting the number of care levels at each unit, i.e. primary care, secondary care, and tertiary care. Similar to the previous dimension, we believe that this way of measuring, although correct, is less meaningful for large hospitals, and that the focus configuration is more aptly captured by measuring focus as emphasis on the dominant care level. In the SLL case, we calculated this emphasis based on the allocation of the highly specialised surgical interventions, since these make up the most well-defined part of tertiary care (Inquiry into Highly Specialised
Care 2015). Hence, the sixth row in Table 4 first shows the overall number of surgeries at each unit (for NKS, 110373; for Sabbatsberg, 15,236; and for Danderyd hospital, 87,146). Then the table shows the number of highly specialised surgeries for each of the three units, which indicates an emphasis on tertiary care, as defined by the Swedish National Board of Health and Welfare as part of the Inquiry into Highly Specialised Care (2015, pp. 255-259). For NKS, the number of highly specialised surgeries is 3406; for Sabbatsberg, 0; and for Danderyd hospital, 360. The highly specialised surgeries as a percentage of all surgeries is then calculated for the three units (for NKS, 3.1\%; for Sabbatsberg, $0 \%$; and for Danderyd hospital, $0.41 \%$ ) and compared to the overall percentage of $0.55 \%$ for SLL as a whole (in all SLL units 6227 highly specialised surgeries are performed annually out of a total number of $1,137,175$ surgeries). NKS is much above the SLL average and thus emphasise tertiary care; the unit performs $55 \%$ of all highly specialised surgeries in the region but only $9.5 \%$ of remaining surgeries. The minimum value, representing highest possible focus on tertiary care, is calculated by allocating $100 \%$ of the highly specialised surgeries within SLL to the same unit (NKS) which would amount to 5.6\%. Sabbatsberg and Danderyd hospital perform less highly specialised surgery than the SLL average and thus have an emphasis on secondary care. The minimum value, representing highest possible focus on secondary care, corresponds to $0 \%$ highly specialised surgeries (i.e. that all surgeries are secondary care level). For all units, the maximum value that represents the lowest possible focus is a split in line with the SLL average, i.e. $0.55 \%$.

After assessing the degree of focus in all six dimensions for the three example units, the resulting values have been normalised to the range of 0 to 1 (see Table 5). The purpose of normalisation is to simplify plotting of values in the spider chart in Fig. 2, and also to facilitate interpretation and comparison between focus values in the different dimensions. However, it is possible to plot the original focus values in Table 4 in the spider chart without normalisation, using different scales on each of the six axes, i.e. scales ranging from the minimum values (stated in Table 4) in the chart centre to the maximum values at the outer edges. The data was normalised using the min-max method, with the minimum and maximum values as defined in Table 4 and with 0 meaning the highest potential degree of focus and 1 meaning the lowest potential degree of focus:

Normalised focus value $=($ focus value - minimum $) /($ maximum - minimum $)$ 


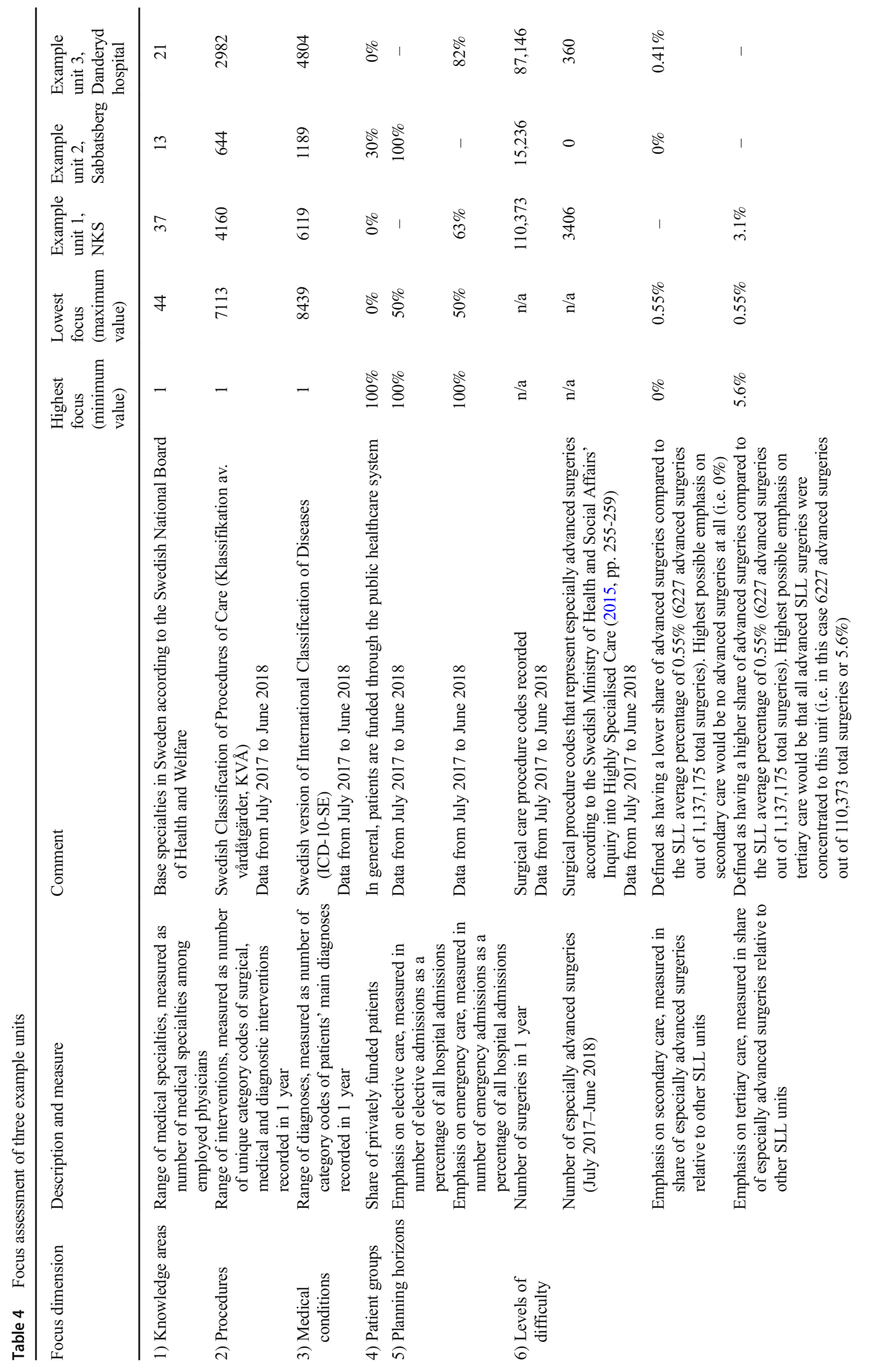


Table 5 Normalised focus assessment of three example units

\begin{tabular}{llll}
\hline Focus dimension & $\begin{array}{l}\text { Example unit 1, } \\
\text { NKS }\end{array}$ & $\begin{array}{l}\text { Example unit 2, } \\
\text { Sabbatsberg }\end{array}$ & $\begin{array}{l}\text { Example unit 3, } \\
\text { Danderyd hospital }\end{array}$ \\
\hline 1) Knowledge areas & 0.84 & 0.28 & 0.47 \\
2) Procedures & 0.58 & 0.09 & 0.42 \\
3) Medical conditions & 0.73 & 0.14 & 0.57 \\
4) Patient groups & 1.00 & 0.70 & 1.00 \\
5) Planning horizons & 0.74 & 0.00 & 0.36 \\
6) Levels of difficulty & 0.50 & 0.00 & 0.75 \\
\hline
\end{tabular}

Open Access This article is distributed under the terms of the Creative Commons Attribution 4.0 International License (http:// creativecommons.org/licenses/by/4.0/), which permits unrestricted use, distribution, and reproduction in any medium, provided you give appropriate credit to the original author(s) and the source, provide a link to the Creative Commons license, and indicate if changes were made.

Publisher's Note Springer Nature remains neutral with regard to jurisdictional claims in published maps and institutional affiliations.

\section{References}

Barratt M, Choi TY, Li M (2011) Qualitative case studies in operations management: trends, research outcomes, and future research implications. J Oper Manag 29:329-342

Berry WL, Klompmaker JE, Bozarth CC, Hill TJ (1991a) Factory focus: segmenting markets from an operations perspective. J Oper Manag 10:363-387

Berry WL, Klompmaker JE, McLaughlin CP, Hill T (1991b) Linking strategy formulation in marketing and operations: empirical research. J Oper Manag 10:294-302

Bohmer RMJ (2009) Designing care: aligning the nature and management of health care. Harvard Business Publishing, Boston

Boyer KK, Pronovost P (2010) What medicine can teach operations: what operations can teach medicine. J Oper Manag 28:367-371

Boyer KK, Bozarth C, McDermott C (2000) Configurations in operations: an emerging area of study. J Oper Manag 18:601-604

Bozarth CC (1993) A conceptual model of manufacturing focus. Int J Oper Prod Manag 13:81-92

Bozarth C, McDermott C (1998) Configurations in manufacturing strategy: a review and directions for future research. J Oper Manag 16: 427-439

Bredenhoff E, van Lent W, van Harten W (2010) Exploring types of focused factories in hospital care: a multiple case study. BMC Health Serv Res 10:154

Capkun V, Messner M, Rissbacher C (2012) Service specialization and operational performance in hospitals. Int J Oper Prod Manag 32: 468-495

Christensen CM, Grossman JH, Hwang J (2009) The innovator's prescription: a disruptive solution for health care. McGraw-Hill, New York

Clark JR (2012) Comorbidity and the limitations of volume and focus as organizing principles. Med Care Res Rev 69:83-102

Clark JR, Huckman RS (2012) Broadening focus: spillovers, complementarities, and specialization in the hospital industry. Manag Sci 58:708-722
Davidow WH, Uttal B (1989) Services companies: focus or falter. Harv Bus Rev 67:77-85

Davis K, Stremikis K, Squires D, Schoen C (2014) Mirror, mirror on the wall, 2014 update: how the U.S. health care system compares internationally. The Commonwealth Fund. http://www. commonwealthfund.org/publications/fund-reports/2014/jun/mirrormirror-wall-2014-update-how-us-health-care-system. Accessed 11 July 2018

De Regge M, Gemmel P, Verhaeghe R, Hommez G, Degadt P, Duyck P (2015) Aligning service processes to the nature of care in hospitals: an exploratory study of the impact of variation. Oper Manag Res 8: $32-47$

Eissens van der Laan MR, van Offenbeek MAG, Broekhuis H, Slaets JPJ (2014) A person-centred segmentation study in elderly care: towards efficient demand-driven care. Soc Sci Med 113:68-76

Fetter RB, Freeman JL (1986) Diagnosis related groups: product line management within hospitals. Acad Manag Rev 11:41-54

Hawthorne J (1993) Bayesian induction is eliminative induction. Philos Top 21:99-138

Hayes RH, Pisano GP (1996) Manufacturing strategy: at the intersection of two paradigm shifts. Prod Oper Manag 5:25-41

Herzlinger RE (1997) Market-driven health care: who wins, who loses in the transformation of America's largest service industry. AddisonWesley, Reading

Heskett JL (1986) Managing in the service economy. Harvard Business Publishing, Boston

Hopp WJ, Lovejoy WS (2012) Hospital operations: principles of high efficiency health care. Pearson FT Press, New York

Hyer NL, Wemmerlöv U, Morris JA Jr (2009) Performance analysis of a focused hospital unit: the case of an integrated trauma center. J Oper Manag 27:203-219

Inquiry into Highly Specialised Care (2015) Träning Ger färdighet. Koncentrera vården för patientens bästa [Practice makes perfect: concentration of care in the best interest of the patient]. (SOU 2015:98). Ministry of Health and Social Affairs, Stockholm

Johnston R (1996) Achieving focus in service organisations. Serv Ind J $16: 10-20$

Joustra P, van der Sluis E, van Dijk N (2010) To pool or not to pool in hospitals: a theoretical and practical comparison for a radiotherapy outpatient department. Ann Oper Res 178:77-89

Ketokivi M, Choi T (2014) Renaissance of case research as a scientific method. J Oper Manag 32:232-240

Krefting L (1991) Rigor in qualitative research: the assessment of trustworthiness. Am J Occup Ther 45:214-222

Law KS, Wong C-S, Mobley WH (1998) Toward a taxonomy of multidimensional constructs. Acad Manag Rev 23:741-755

Lynn J, Straube BM, Bell KM, Jencks SF, Kambic RT (2007) Using population segmentation to provide better health care for all: the "bridges to health" model. Milbank Q 85:185-208 
McDermott CM, Stock GN (2011) Focus as emphasis: conceptual and performance implications for hospitals. J Oper Manag 29:616-626

McDermott C, Stock G, Shah R (2011) Relating focus to quality and cost in a healthcare setting. Oper Manag Res 4:127-137

McLaughlin CP, Yang S, van Dierdonck R (1995) Professional service organizations and focus. Manag Sci 41:1185-1193

Miles MB, Huberman AM, Saldaña J (2014) Qualitative data analysis: a methods sourcebook. Sage, Los Angeles

Mukherjee A, Mitchell W, Talbot FB (2000) The impact of new manufacturing requirements on production line productivity and quality at a focused factory. J Oper Manag 18:139-168

OECD (2013) OECD reviews of health care quality: Sweden 2013. OECD Publishing, Paris

Peltokorpi A, Linna M, Malmström T, Torkki P, Lillrank PM (2016) Five focus strategies to organize health care delivery. Int J Health Care Qual Assur 29:177-191

Pieters A, van Oirschot C, Akkermans H (2010) No cure for all evils. Int J Oper Prod Manag 30:1112-1139

Porter ME, Teisberg EO (2006) Redefining health care: creating valuebased competition on results. Harvard Business Publishing, Boston
Sampson SE, Froehle CM (2006) Foundations and implications of a proposed unified services theory. Prod Oper Manag 15:329-343

Schmenner RW, Swink ML (1998) On theory in operations management. J Oper Manag 17:97-113

Silvestro R (1999) Positioning services along the volume-variety diagonal: the contingencies of service design, control and improvement. Int J Oper Prod Manag 19:399-421

Silvestro R, Fitzgerald L, Johnston R, Voss C (1992) Towards a classification of service processes. Int J Serv Ind Manag 3:62-75

Singh KCD, Terwiesch C (2011) The effects of focus on performance: evidence from California hospitals. Manag Sci 57:1897-1912

Skinner W (1974) The focused factory. Harvard Bus Rev 52:113-120

van Dierdonck R, Brandt G (1988) The focused factory in service industry. Int J Oper Prod Manag 8:31-38

Voss C, Tsikriktsis N, Frohlich M (2002) Case research in operations management. Int J Oper Prod Manag 22:195-219

Zonderland ME, Boucherie RJ, Litvak N, Vleggeert-Lankamp CLAM (2010) Planning and scheduling of semi-urgent surgeries. Health Care Manag Sci 13:256-267 